\title{
A Capacitively Loaded PIFA for Compact Mobile Telephone Handsets ${ }^{\dagger}$
}

\author{
Corbett R. Rowell $\stackrel{\frac{1}{*}}{*}$, and R. D. Murch (Member IEEE) \\ Department of Electrical and Electronic Engineering \\ The Hong Kong University of Science \&5 Technology \\ Clear Water Bay, Kowloon, HONG KONG \\ Revised July $30^{\text {th }}, 1996$
}

\begin{abstract}
A capacitively loaded planar inverted-F antenna (PIFA) is proposed and studied. It is found that the capacitive load reduces the resonance length of the PIFA from $\lambda / 4$ to less than $\lambda / 8$. A design with a bandwidth of $178 \mathrm{MHz}$ centered at $1.8 \mathrm{GHz}$ is provided to demonstrate that compact antennas for mobile telephone handsets can be constructed using this approach. The finite difference time domain method is used in the study and experimental verification is also provided.
\end{abstract}

$\dagger$ Supported by the Hong Kong research grant council, Project HKUST788/96E and CRC93/94.EG01.

$\ddagger$ Corresponding Author. Email: phcrr@ee.ust.hk. Phone: (+852) 2358 7082. Fax: (+852) 23581485. 


\section{INTRODUCTION}

In recent years, the demand for compact mobile telephone handsets has grown. Handsets the size of a deck of cards have begun appearing in the market and as the demand for increased electronic mobility grows, the need for smaller handsets will most likely increase. The handset size, however, is limited by the battery and the size of the antenna. In addition, the need to employ antenna diversity or adaptive antennas on the mobile telephone handset to improve receiver performance increases the need for small antennas. In the past few years, new designs arising from the planar inverted $\mathrm{F}$ antenna (PIFA) have merited attention in addressing these demands [1]-[4]. PIFAs are compact (approximately $\lambda / 4$ length with a low profile) and can further be optimized by using strategically placed loads.

This paper investigates the feasibility of utilizing a PIFA with a capacitive load to reduce the overall length to less than $\lambda / 8$ (without using dielectrics) for a mobile telephone handset. The standard coax feed is also replaced by a capacitive feed [10] to allow further manipulation of the impedance curves. The capacitively loaded antenna designs are compared to the PIFAs without the capacitive load or feed. Employing the finite difference time domain (FDTD) method [5]-[8], the properties of the antenna system are explored. Experimental results validate the FDTD simulations. Furthermore, a transmission line model is also developed that allows the analysis to be performed quickly for design purposes.

In Section II we describe the proposed antenna structure. Computational results are presented in Section III while a design suitable for Personal Communication System (PCS) applications is provided in Section IV. Experimental results of a prototype based on this design are also provided. An approximate transmission line model for the antenna is introduced in Section $\mathrm{V}$ and conclusions are provided in Section VI where rules for designing antennas based on our structure are given.

\section{Structure}

The antenna structure is based around a PIFA but incorporates a capacitive load and feed as shown in figure 1 . The capacitive load is formed by folding the open end of the PIFA 
toward the ground plane and adding a plate (parallel to the ground plane) to produce a parallel plate capacitor for the load. The capacitive feed is constructed by terminating the inner conductor of a coaxial to a conducting plate. The conducting plate electromagnetically couples to the radiating top plate. The antenna is mounted on a small conducting box of dimensions $80 \mathrm{~mm} \times 40 \mathrm{~mm} \times 10 \mathrm{~mm}$, producing a handset suitable for mobile telephone applications (figure 1).

For the purpose of analyzing the performance of the antenna it is useful to specify the dimensions of a test antenna geometry. For the test geometry the radiating top plate dimensions are $\left(l_{p i f a}, w_{p i f a}\right)=(25 \mathrm{~mm}, 10 \mathrm{~mm})$ with a height $h_{p i f a}=5 \mathrm{~mm}$. The capacitive load plate dimensions are $\left(w_{c a p}, w_{\text {pifa }}\right)=(4 \mathrm{~mm}, 10 \mathrm{~mm})$ and is separated from the ground plane by $d_{c a p}=0.5 \mathrm{~mm}$ while the capacitive feed dimensions are $\left(l_{c f}, w_{c f}\right)=(23 \mathrm{~mm}, 10 \mathrm{~mm})$ and is separated from the top plate by $d_{c f}=2.5 \mathrm{~mm}$. The short pin is placed equidistant between either side of the top plate. The antenna is situated on the handset such that the short pin and the feed are equi-distant from the handset sidewalls. In Section IV the test antenna dimensions are modified to produce an antenna suitable for PCS applications.

\section{Computational Results}

To obtain computational results for our antenna design FDTD simulations were performed. To perform the simulations we utilized an FDTD code that was a modified version of the publicly available code located in [6]. The code has been altered to allow antenna simulations and an integral near-field to far-field transformation is implemented [7]. The source is a Gaussian derivative of the form:

$$
\left.V_{\text {source }}=(-2 \alpha(\tau-\beta \Delta t) \Delta t) e^{\left(-\alpha(\tau-\beta \Delta t)^{2}\right)}\right)
$$

where $\beta$ sets the pulse width, $\tau$ is the time delay of the pulse, and $\alpha$ sets the source value at truncation of the pulse. To prevent numerical resonance, the source is modeled as a voltage source in series with a resistor to absorb the stray current. Generated results have 
been compared with impedance and far-field calculations presented in [2], [6]- [9], and the canonical dipole radiator, validating the accuracy of our FDTD program.

Without a capacitive load or feed, the resonant frequency of the test antenna geometry (using our FDTD code) is $2.48 \mathrm{GHz}$. To achieve a resonance at $1.8 \mathrm{GHz}$ (for PCS applications) with an unloaded PIFA, the overall length would need to be approximately $42 \mathrm{~mm}$. To try and reduce this length we investigate adding a capacitive load and feed to the PIFA structure.

\section{A. Capacitive Load}

The effect of the capacitive load on the antenna performance is investigated by varying the capacitive load's width $w_{\text {cap }}$ and plate separation $d_{c a p}$ while keeping the remaining dimensions of the antenna geometry fixed as for the test geometry defined in Section II.

In figures 2 and 3 we present the impedance and VSWR characteristics of the antenna for several $w_{\text {cap }}$. By utilizing a parallel plate capacitor approximation such that $C \propto w_{\text {cap }} / d_{\text {cap }}$ we can notionally equate a particular $w_{c a p}$ and $d_{c a p}$ to a load capacitance. It can then be observed that as the capacitive load increases (increasing $w_{\text {cap }} / d_{\text {cap }}$ ), the resistive and reactance peaks in antenna impedance increase while the width of these peaks contract (figure 2), thereby decreasing the bandwidth of the structure (figure 3 ). From these results it is realized that the capacitive load reduces the resonant frequency but at the expense of bandwidth and good matching.

In figure 4 we plot the resonant frequency (which we define as where the phase of the input impedance is equal to zero) for various $d_{\text {cap }}$ when $w_{\text {cap }}$ is varied between 0.5 and $8 \mathrm{~mm}$. It can be observed that the larger capacitive loads offer reductions in resonant frequency of nearly $1 \mathrm{GHz}$ compared to the unloaded PIFA (figure 4). In the next section we show how matching may be improved by altering the dimensions of the capacitive feed to utilize these potential reductions in resonant frequency. 


\section{B. Capacitive Feed}

To improve the impedance characteristics of our antenna we utilize a capacitive feed. The capacitive feed (also known as non-contact feed) has received extensive attention [10]- [13]. By changing three parameters - the area of the feed plate, the separation from the radiating top plate, and probe placement on the feed plate-the antenna designer has control over the resonance properties of the antenna ([10] contains results on each change). Here we concentrate on the adjustment of the first two parameters, area and plate separation, to provide the necessary impedance characteristics of our antenna to enable it to be employed in PCS applications. However probe placement on the capacitive feed is also known to affect impedance characteristics (documented in [10]) and this may be used for further design flexibility in other applications.

In figures 5 and 6 , we present the impedance characteristics for two values of plate separation $d_{c f}$ and plate area $A_{c f}=w_{c f} \times l_{c f}$ when the remaining antenna dimensions are fixed as for the test geometry defined in Section II. As the distance $d_{c f}$ increases, the magnitude of the local maxima in resistance decreases (figure 5), otherwise leaving the general shape of the resistance curve unchanged. The increased distance $d_{c f}$ reduces the local maxima of the reactive curve and translates the curve vertically downward. The area $A_{c f}$, however, only affects the reactance curve (figure 6). As $A_{c f}$ increases, the reactance curve vertically shifts upward. The resistance is unaffected by increases in the area $A_{c f}$ unless it becomes large enough to cause the capacitive feed to become unshielded by the top plate. That is the corresponding feed plate width or length is greater than that of the top plate or when it is offset from the top plate so part of it is unshielded. This is because the combination of the radiating top plate and the feed plate will then act together as a larger radiating plate reducing the resonant frequency and affecting input resistance [10].

Utilizing these two properties one can manipulate the impedance curves and adjust the resonant characteristics. Although the horizontal placement along the frequency axis is unchanged, the vertical adjustment of the reactance allows the designer to "choose" a res- 
onance frequency (where the reactance $=0$ ) in a $200-300 \mathrm{MHz}$ range. For the proposed capacitively loaded antenna, the distance $d_{c f}=2.5 \mathrm{~mm}$ reduces the magnitude of the resistance from $250 \Omega$ to $50-100 \Omega$. The area $A_{c f}=23 \mathrm{~mm}$ x $10 \mathrm{~mm}$ (maximum area allowed without radiation effects), places the resonant frequency near the start of the resonance range.

\section{Far Field}

The far fields are relatively unaffected by the addition of the electromagnetically coupled feed since the feed structure does not contribute to the radiating energy of the system [10]. The capacitive load, however, has minor effects on the far field patterns (figure 7 ). In the H-plane, the capacitively loaded antenna has eliminated the two notches in the antenna pattern. The E-plane radiation pattern becomes more uniform, retaining a notch in the lower left due to the handset.

\section{Proposed PCS Antenna Design}

Using the results presented in the previous section, we design the minimum volume capacitive loaded PIFA antenna for DCS 1800 operation (frequency band 1.71 to $1.88 \mathrm{GHz}[15]$ ). To perform this we take the results from our test geometry and scale the dimensions appropriately so that a resonant frequency of $1.795 \mathrm{GHz}$ is obtained for each capacitive load configuration. We then calculate the volume and bandwidth of each of these designs and choose the design with the minimum volume which meets DCS 1800 bandwidth specifications.

The volume as a function of the bandwidth ( $V S W R<2$ for a $50 \Omega$ impedance match) is graphed in figure 8 for various capacitive load configurations. Each line in the graph belongs to a set of simulations specified by the distance separating the capacitive load plate from ground $\left(d_{\text {cap }}\right)$ while the width $w_{c a p}$ is varied. As the capacitive width $w_{c a p}$ increases, the capacitive load increases. Therefore, the volume gets smaller as $w_{\text {cap }}$ increases; and as a result, the bandwidth also decreases. Limited by the DCS 1800 bandwidth of $170 \mathrm{MHz}$, a 
design with a total volume of $640 \mathrm{~mm}^{3}$ is found. Figure 9 shows the resulting antenna with a capacitive load of $d_{\text {cap }}=0.4 \mathrm{~mm}, w_{\text {cap }}=0.5 \mathrm{~mm}$ (a capacitance of $1.478 \mathrm{pF}$ ). The antenna has a bandwidth of $178 \mathrm{MHz}$, centered at $1.795 \mathrm{GHz}$. Prototyping this antenna and using an HP8510c vector network analyzer to measure VSWR, experimental results have been obtained and these are presented in figure 10 .

The radiation efficiency of the antenna has not been measured or analyzed however we can highlight some relevant points. The power loss mechanisms occurring in the antenna will be directly related to the current flow over the finite conductivity plates since dielectric and other loss mechanisms are not present. Consequently, an estimate of the comparative efficiency of this antenna to a conventional PIFA can be found from comparing the current distributions. In the capacitively loaded PIFA the current distribution on the top plate will be more uniform and larger in magnitude than in a conventional PIFA because of the capacitive load. In addition the current flowing on the capacitive feed plate will also add to the losses. Offsetting these losses however will be the reduced losses from the decrease in length of the capacitively loaded PIFA. Thus we can conclude that the efficiency will likely be slightly less than that of a conventional PIFA.

\section{Transmission Line Model}

Before concluding this paper it is also worthwhile noting that a transmission line model analysis can be applied that usefully approximates some FDTD results we have obtained. Such analysis is useful in the design cycle for our antenna.

The transmission line model treats the PIFA as two transmission lines of lengths $l_{1}, l_{2}$ respectively between the feed and the shorted and open ends of the PIFA as illustrated in figure 12. The capacitor load is modeled as a capacitor load in parallel with a resistive load with radiation resistance $R_{r}$. The capacitive load can be thought of as being equivalent to a length $L_{o c}$ of open circuited transmission line. Adding the physical length $L_{p h y s}, L_{o c}$, and 
the height $H$ of the antenna, resonance of the antenna can be expressed as:

$$
L_{p h y s}+L_{o c}+H=\lambda / 4
$$

Solving for $L_{o c}$ and using the equation $C=L_{o c} / Z_{0} v$ (where $v$ is the speed of the propagating electromagnetic wave inside the antenna and $Z_{0}=50 \Omega$ ), the capacitance as a function of resonant frequency is plotted in figure 11. It can be observed that good agreement with the FDTD results is obtained. It is also worth noting in figure 11 that the upper region of the curve is nearly linear on the log-linear plot.

\section{CONCLUSIONS}

With the addition of a capacitive load we have shown that the dimensions of a PIFA for a given resonant frequency can be significantly reduced. The resultant difficult matching is overcome by a capacitive feed used to manipulate the resistance and reactance curves. The small volume of the resulting antenna design would make it a useful candidate for incorporation into future diversity and adaptive antenna PCS handset designs and a design suitable for DCS 1800 is proposed. A design methodology for capacitive-loaded PIFAs is proposed below:

- Determine approximate constraints on the antenna width, length, and height.

- Run two-three simulations of different capacitive load geometries using FDTD and transmission line analysis.

- Plot $C(f)$ using equation 2 on a log-linear graph.

- Determine slope on log-linear portion, and extrapolate to find the capacitance at the desired frequency.

- Working backwards, determine the appropriate dimensions of the capacitive load.

- Attain a good match by adjusting the capacitive feed parameters as described in [10].

\section{REFERENCES}

[1] J. Fuhl, P. Nowak, and E. Bonek Improved internal antenna for hand-held terminals. Electronic Letters 
$30(22): 1816-1818,1994$.

[2] G. Pederson and J. Andersen. Integrated Antennas for Hand-Held Telephones with Low Absorption. 1994 IEEE 44th Vehicular Technology Conference pages 1537-1541.

[3] T. Taga Analysis of planar inverted-F antennas and antenna design for portable radio equipment, Analysis, Design, and Measurement of Small and Low Profile Antennas. K. Hirasawa and M. Haneishi, Eds. Boston: Artech, 1992, ch. 5.

[4] T. Taga and K. Tsunekawa Performance analysis of a built-in planar inverted F antenna for $800 \mathrm{MHz}$ band portable radio units. IEEE J. Selected Areas Commun. SAC-35: 921-929, June 1987.

[5] K.S. Yee Numerical Solution of Initial Boundary Value Problems Involving Maxwell's Equations in Isotropic Media IEEE Trans. Antennas Propagat. AP-14: 302-307, May 1966.

[6] K. S. Kunz and R. J. Luebbers. The Finite Difference Time Domain Method for Electromagnetics. CRC Press (Boca Raton, FL) 1993.

[7] R.J. Luebbers, K.S. Kunz, M. Schneider, and F. Hunsberger. A Finite-Difference Time-Domain Near Zone to Far Zone Transformation. IEEE Trans. Antennas Propagat., 39(4):429-433, 1991.

[8] R. Luebbers, L. Chen, T. Uno, and S. Adachi. FDTD Calculation of Radiation Patterns, Impedance, and Gain for a Monopole Antenna on a Conducting Box. IEEE Trans. Antennas Propagat., 40(12):1577$1583,1992$.

[9] M.A. Jensen and Y. Rahmat-Samii. Performance Analysis of Antennas for Hand-Held Transceivers Using FDTD. IEEE Trans. Antennas Propagat., 42(8):1106-1113, 1994.

[10] Guy A.E. Vandenbosch and Antoine R. Van de Capelle Study of the Capacitively Fed Microstrip Antenna Element. IEEE Trans. Antennas Propagat. 42(12):1648-1652, 1994.

[11] M.J. Alexander Capacitive matching of microstrip patch antennas. IEE Proceedings H 136(2): 172-174, 1989.

[12] G.A.E. Vandenbosch Capacitive matching of microstrip antennas Electronics Letters 31(18): 1535$1536,1995$.

[13] K. Kagoshima, K. Tsunekawa, and A. Ando Analysis of a planar inverted F antenna fed by electromagnetic coupling. IEEE Antennas and Propagation Society International Symposium. (Cat. No.92CH31781): 1702-1705, 1992 .

[14] Richard C. Johnson Antenna Engineering Handbook Chapter 7: Microstrip Antennas McGraw-Hill: 1993.

[15] M. Mouly and M.B. Pautet The GSM System for Mobile Communications 1992. 

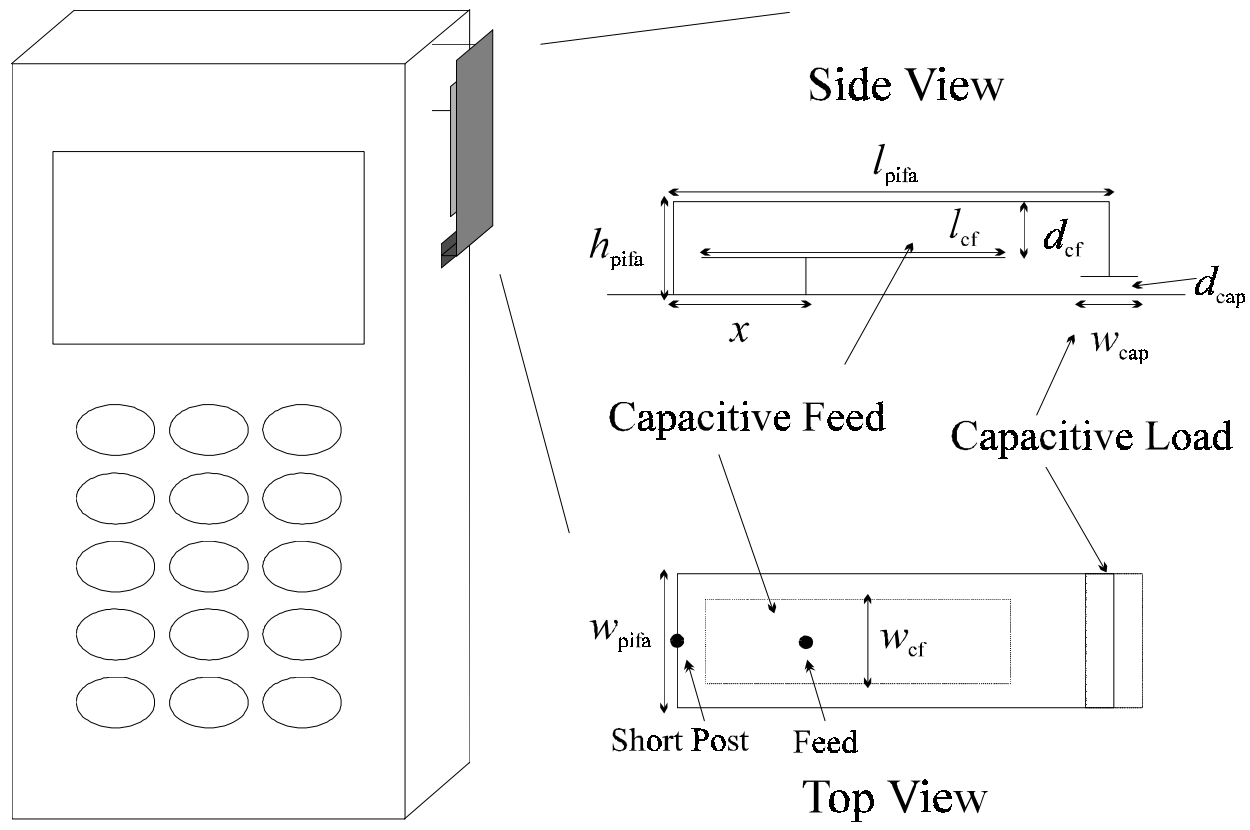

Fig. 1. The capacitively loaded PIFA antenna structure. The dimensions of the top plate (or the transmission plate) are $\left(l_{p i f a}, w_{p i f a}\right)$ while the capacitive load has dimensions $\left(w_{c a p}, w_{p i f a}\right)$ and is separated from the ground plane by $d_{c a p}$. The capacitive feed plate dimensions are $\left(l_{c f}, w_{c f}\right)$ and is separated from the top plate by $d_{c f}$. The handset (on left) is $80 \mathrm{~mm} \times 40 \mathrm{~mm}$ $\mathrm{x} 10 \mathrm{~mm}$.

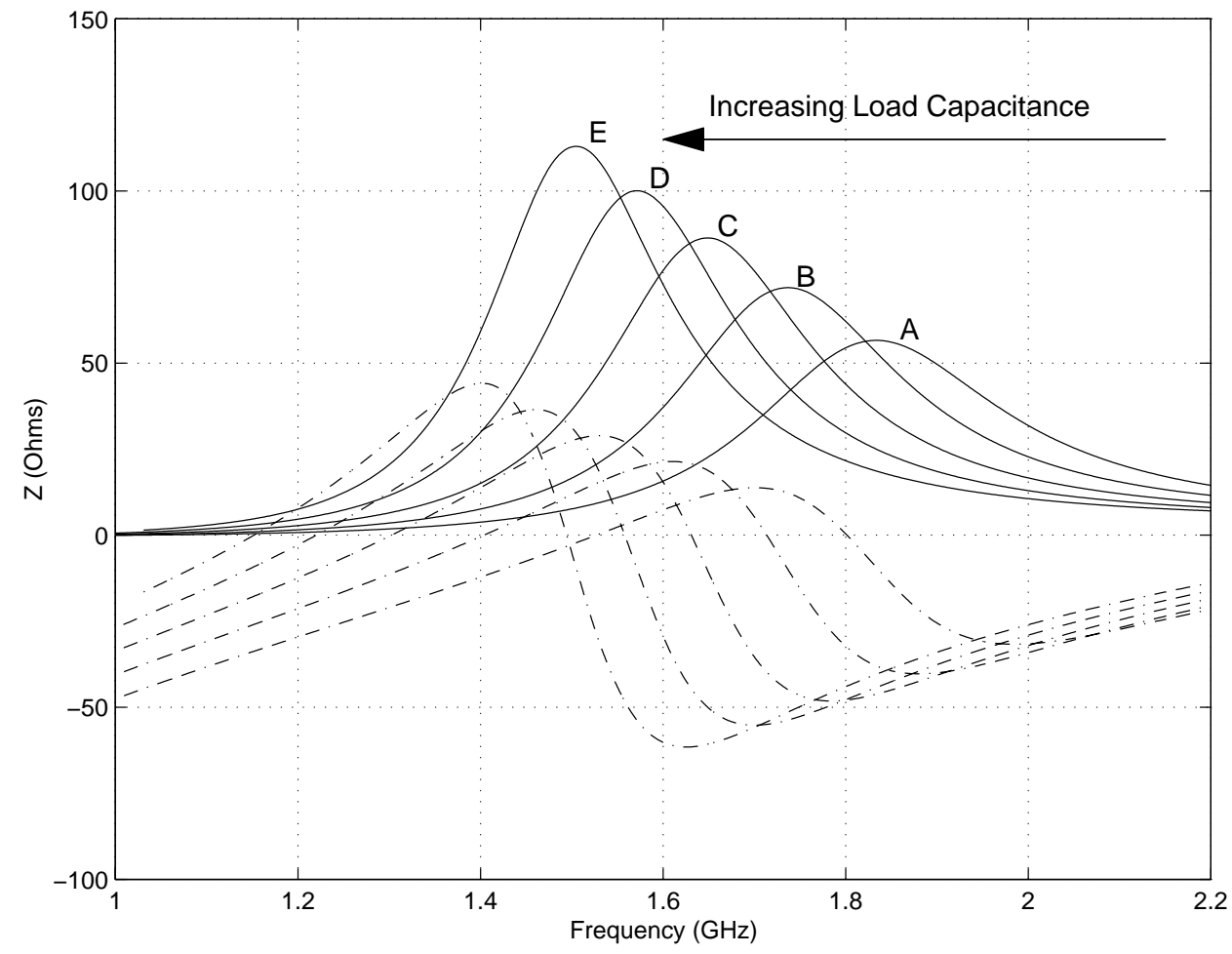

Fig. 2. The solid and dashed lines respectively represent the antenna input resistance and reactance as a function of frequency. Each of the lines $(A, B, C, D, E)$ respectively refers to when the capacitive load dimensions $\left(w_{\text {cap }}, d_{\text {cap }}\right)$ are $(0.5,3),(2,3),(4,3),(6,3),(8,3) \mathrm{mm}$. As the ratio $w_{c a p} / d_{c a p}$ increases (increasing load capacitance), the impedance curves grow larger in magnitude. 


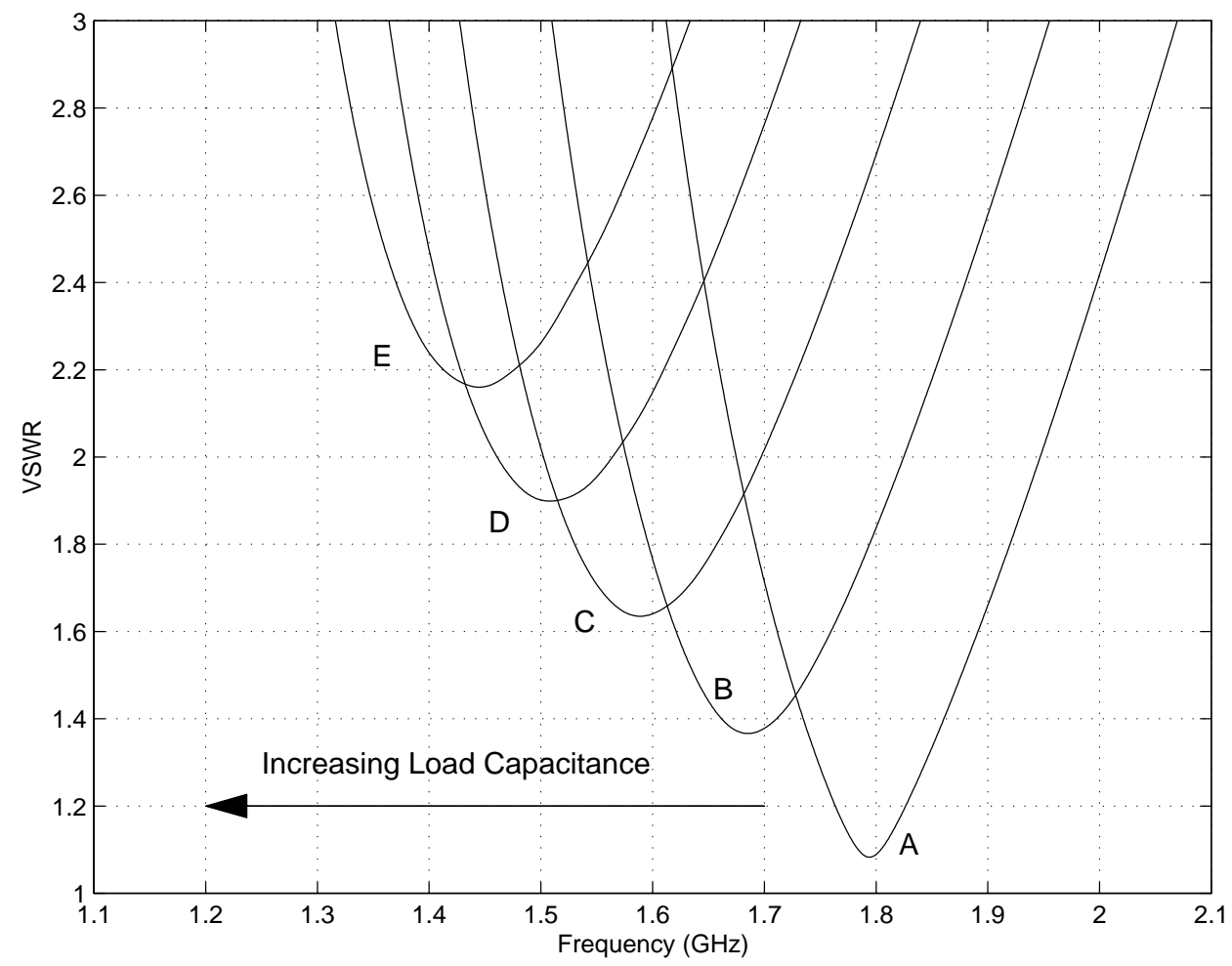

Fig. 3. VSWR (for a $50 \Omega$ match) as a function of frequency. Each of the lines $(A, B, C, D, E)$ refer to the same $\left(w_{c a p}, d_{c a p}\right)$ dimensions as in figure 2

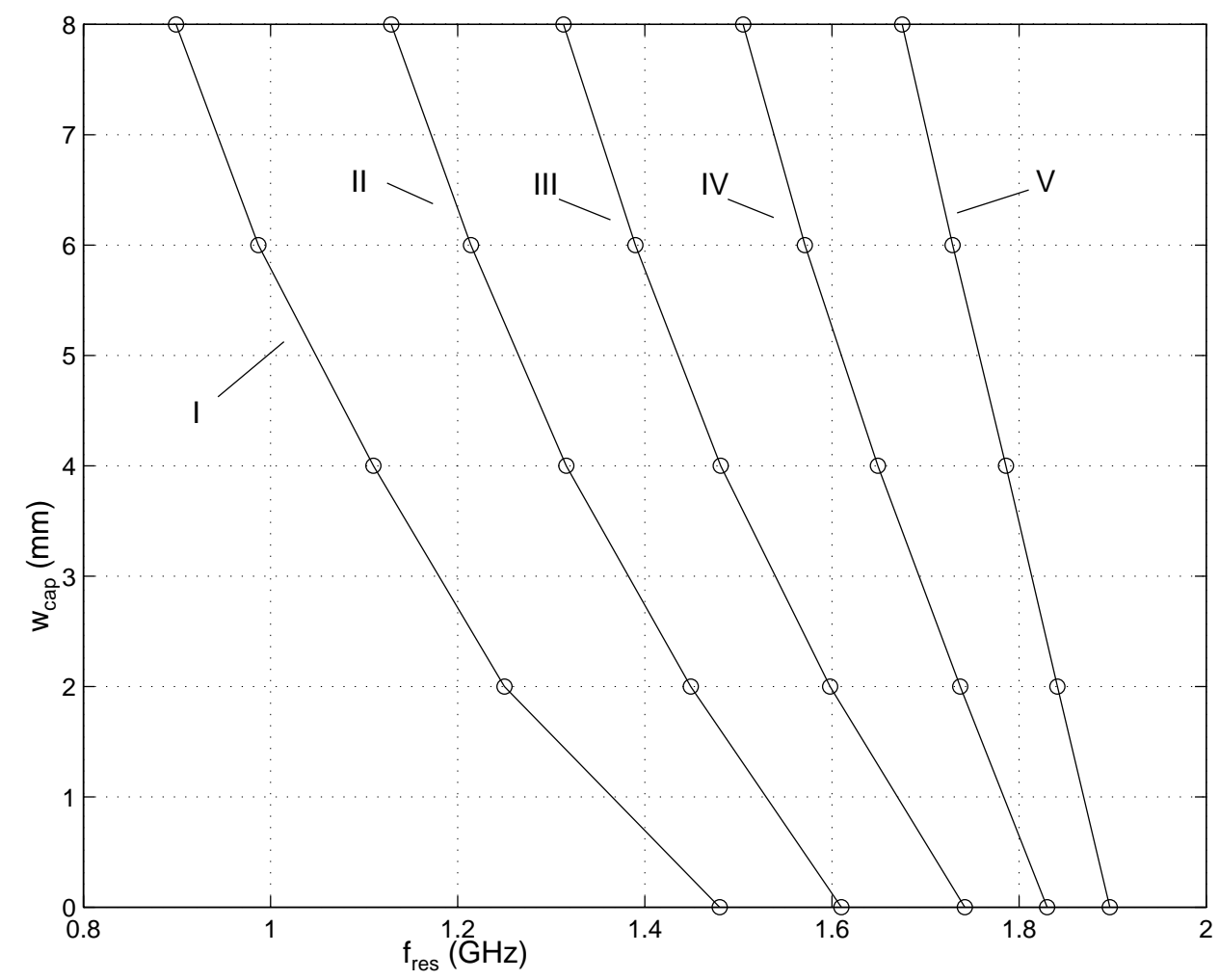

Fig. 4. Capacitive load dimensions $\left(w_{c a p}, d_{c a p}\right)$ as a function of the resonant antenna frequency (defined as where the input reactance is zero). Each of the lines (I-V) refer to $d_{\text {cap }}=(0.5,1.0,2.0,3.0,4.0) \mathrm{mm}$ respectively. 


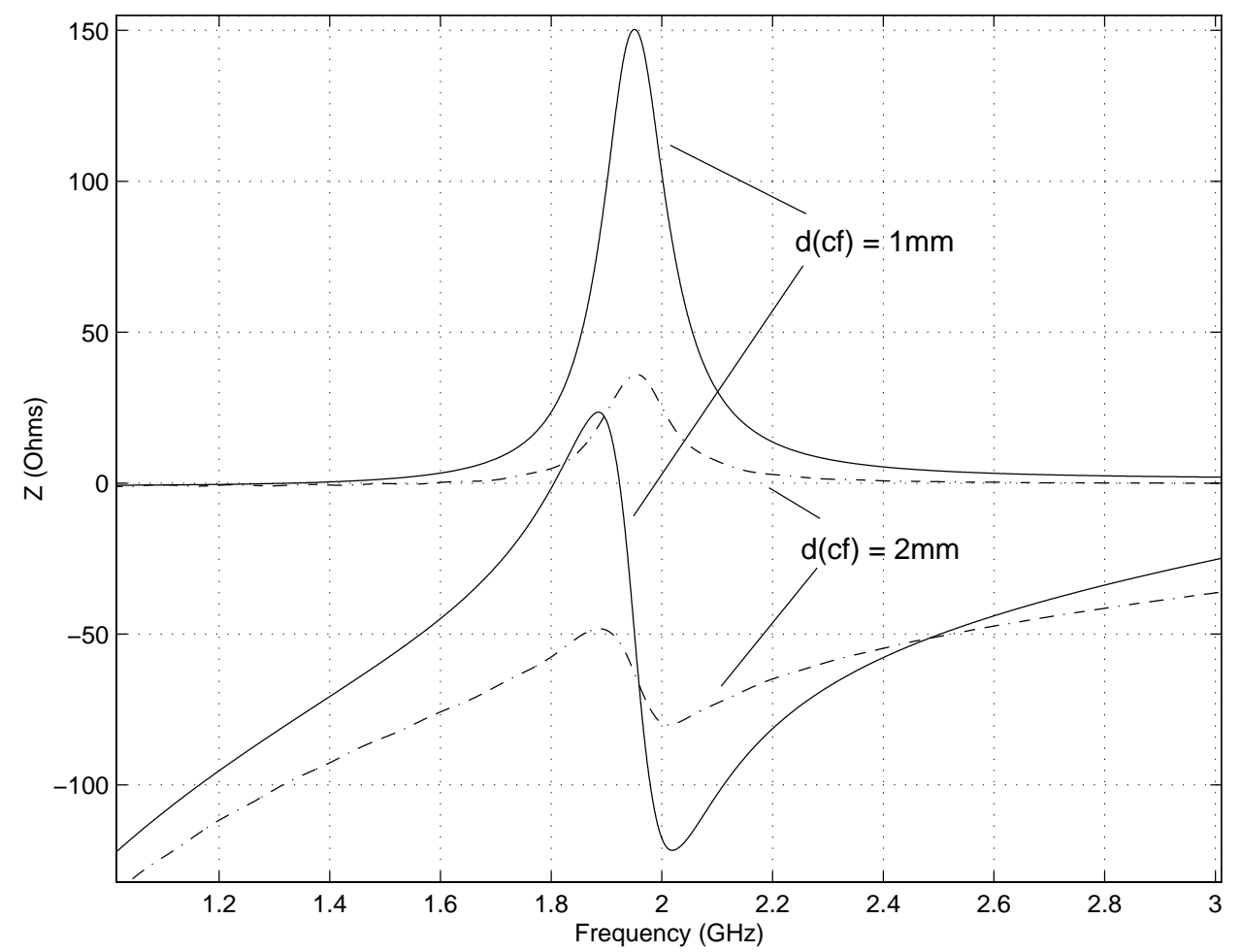

Fig. 5. Input resistance and reactance curves for two feed plate separations. The solid lines are for a separation distance of $d_{c f}=1 \mathrm{~mm}$ and the dotted lines for a separation distance of $d_{c f}=2 m m$. 


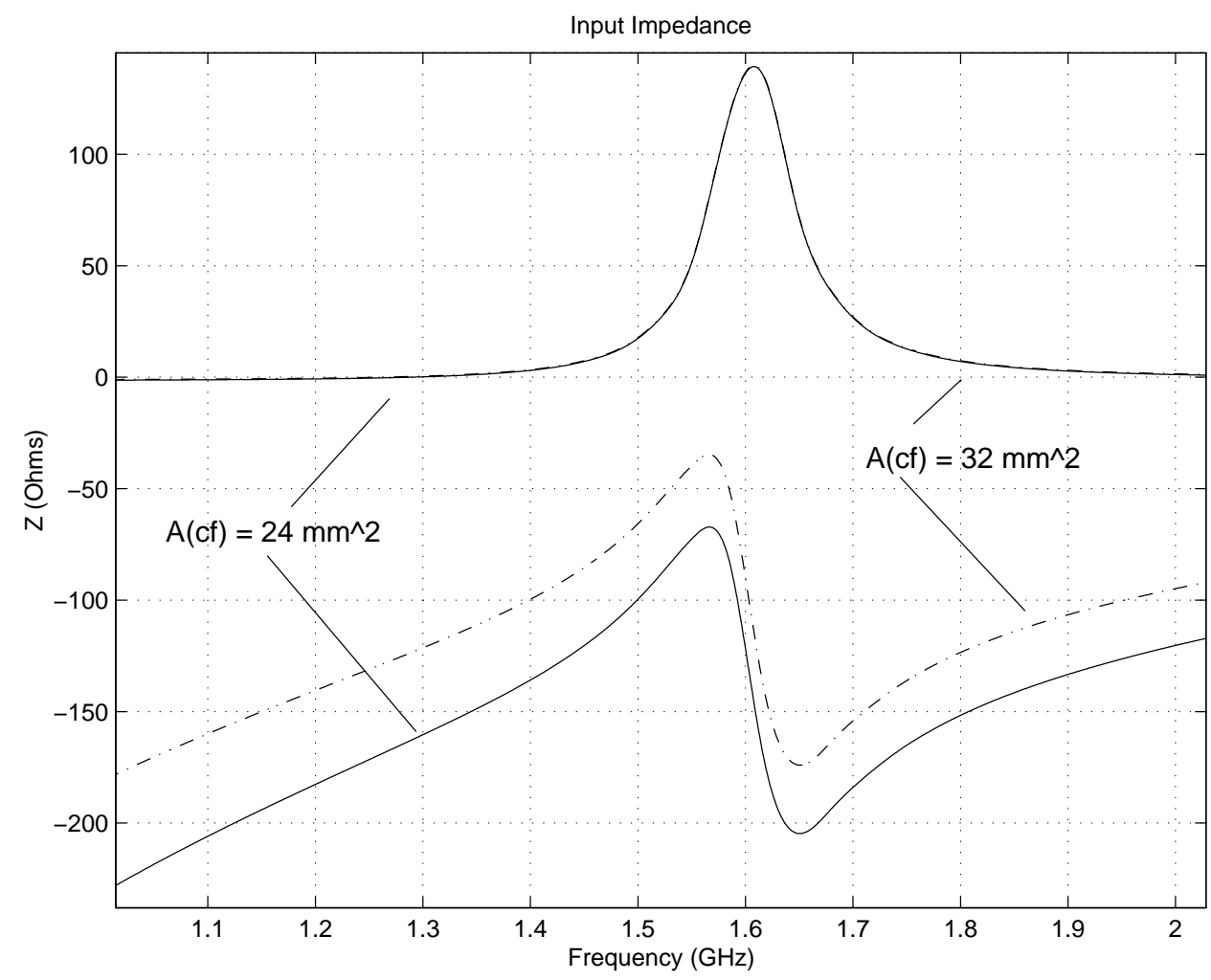

Fig. 6. Input resistance and reactance curves for two feed plate areas. The solid lines are for a capacitive feed plate area of $A_{c f}=24 \mathrm{~mm}^{2}$ and the dotted lines for a capacitive feed plate area of $A_{c f}=32 \mathrm{~mm}^{2}$. (Note: The resistance curves overlay each other)
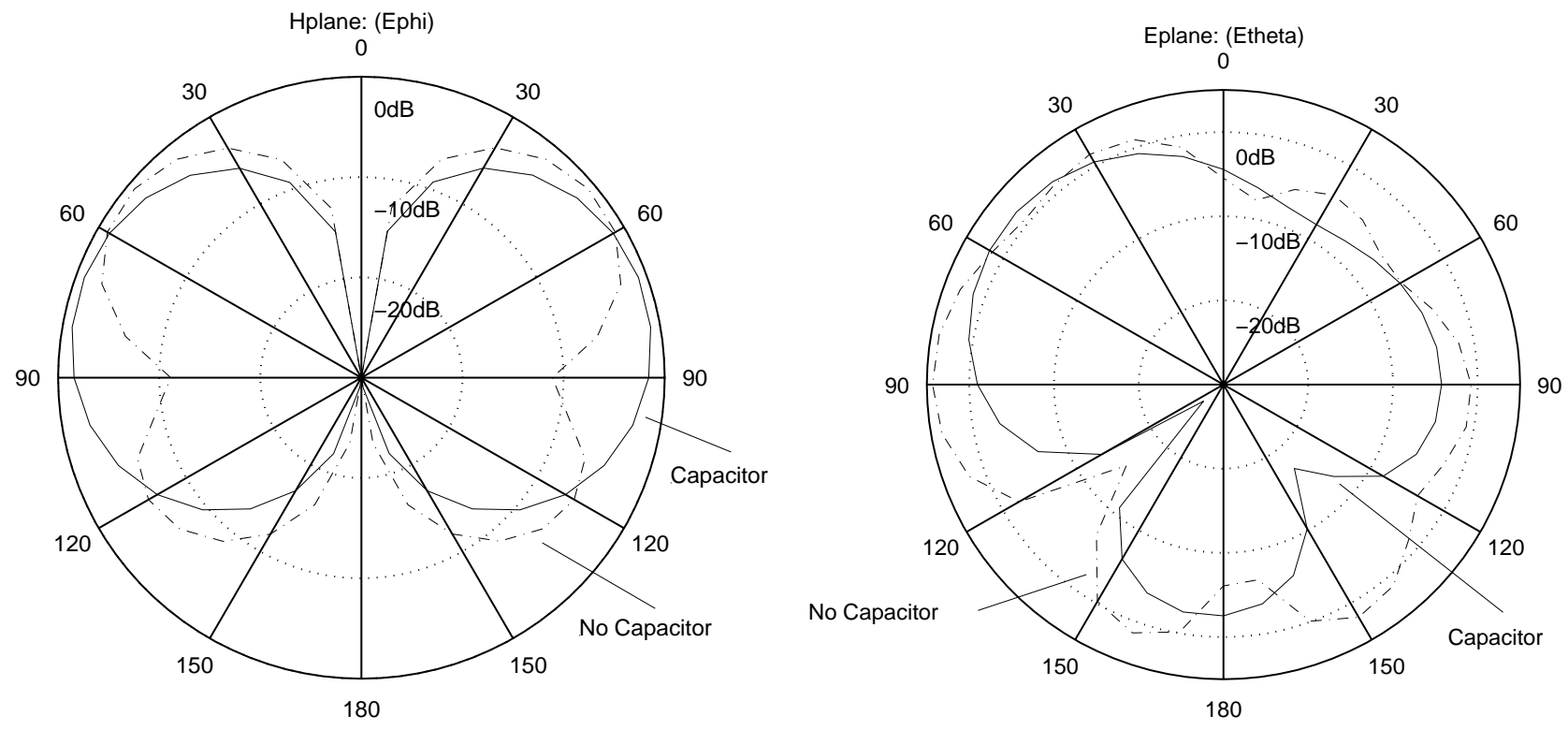

Fig. 7. The solid and dashed lines are respectively the far-field results for the PIFA with and without the capacitive load in $\mathrm{dBi}$. 


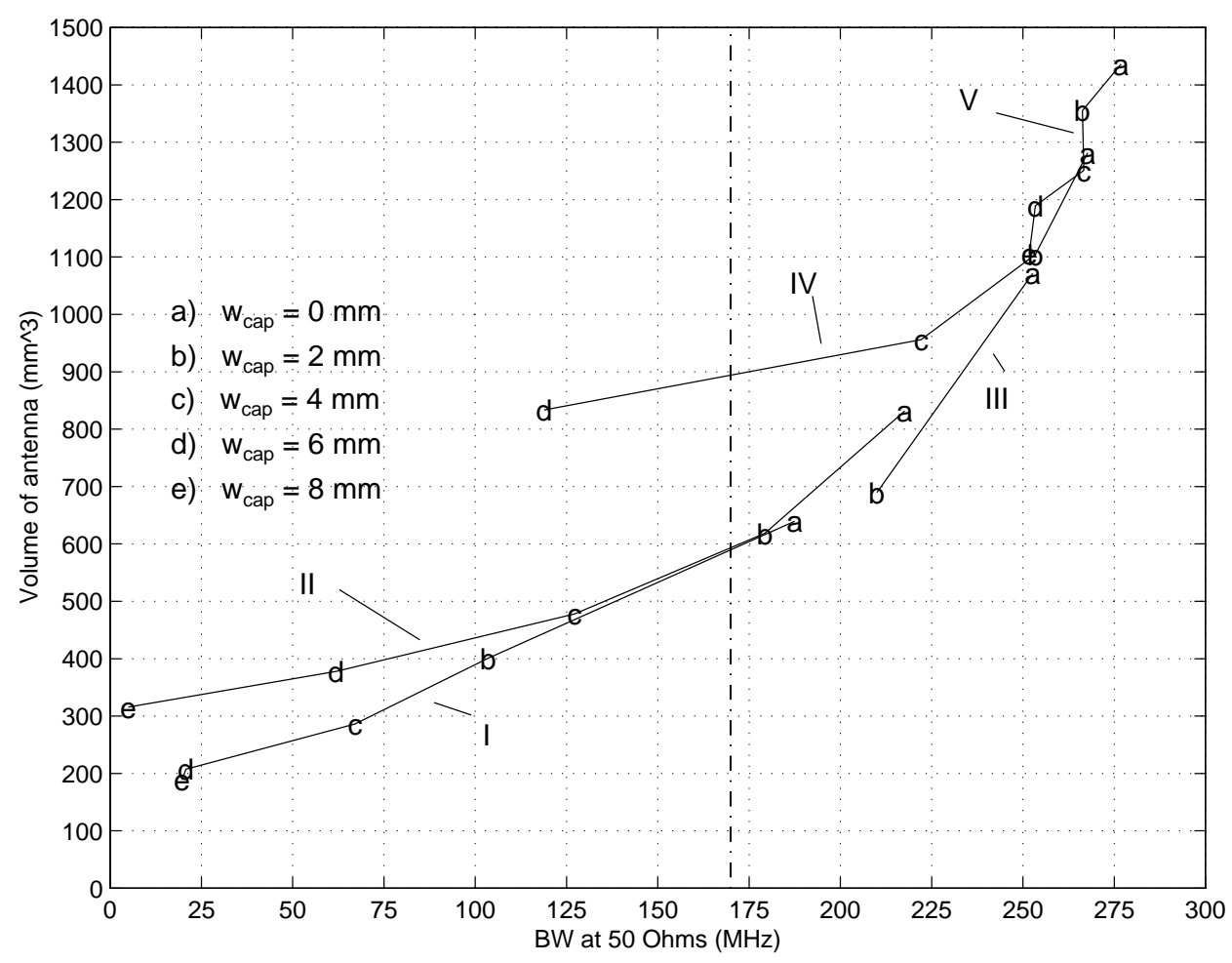

Fig. 8. Volume as a function of the bandwidth ( $V S W R<2$ for a $50 \Omega$ impedance match) for various capacitive load configurations when the resonant frequency is fixed (by scaling the PIFA dimensions) at $1.795 \mathrm{GHz}$. The results (I-V) represent $d_{\text {cap }}=(0.5,1.0,2.0,3.0,4.0) \mathrm{mm}$ when $w_{c a p}$ is varied from $0.0-8 \mathrm{~mm}$ ( $w_{c a p}$ is specified by the letters $a$ to $e$ as listed in the key.)

\section{Side View}
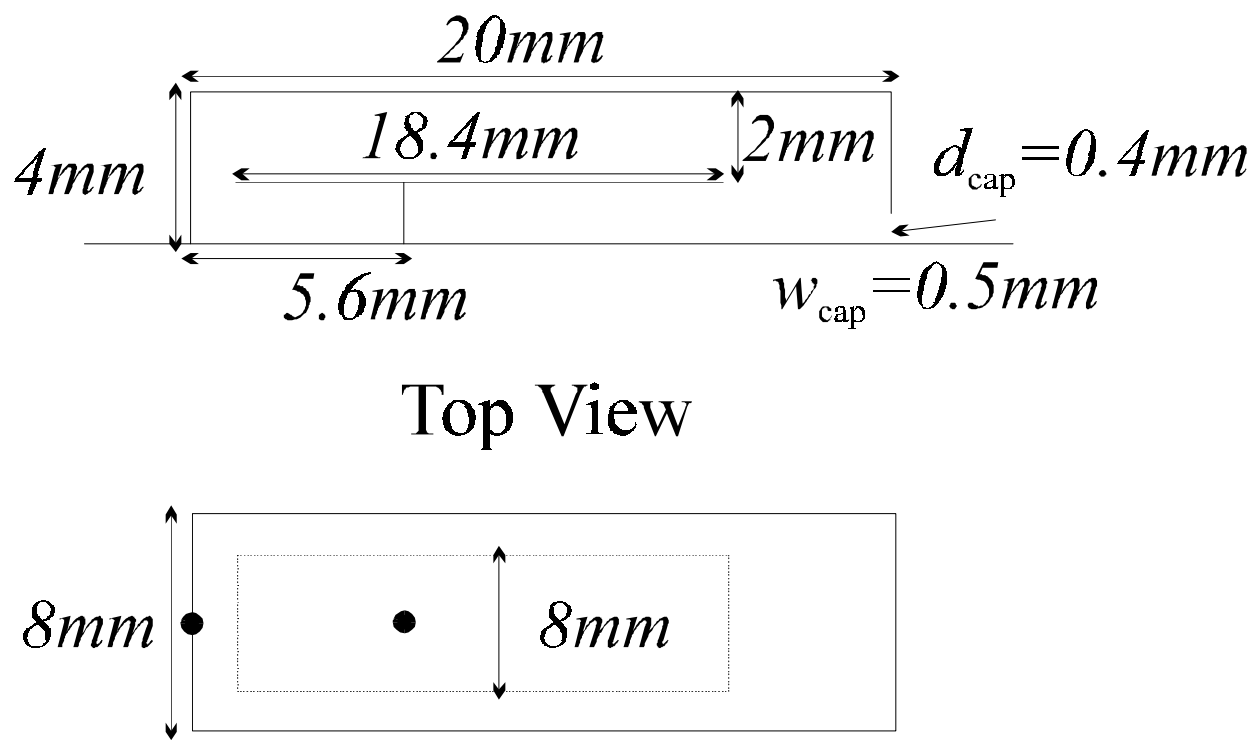

Fig. 9. Capacitive loaded PIFA design suitable for DCS 1800 operation. The total volume is $640 \mathrm{~mm}^{3}$ and the antenna has a bandwidth of $178 \mathrm{MHz}$ centered at $1.795 \mathrm{GHz}$. 


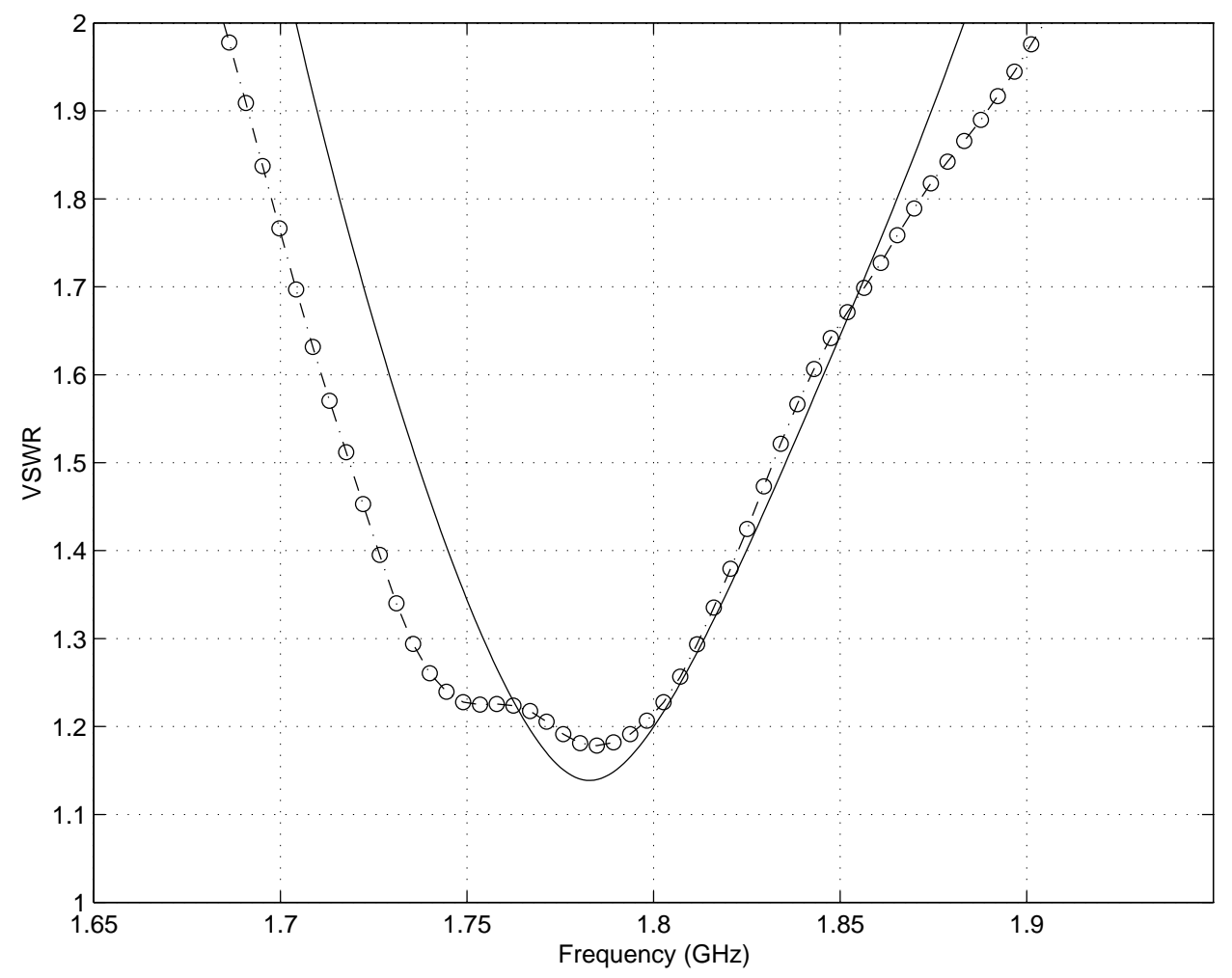

Fig. 10. Experimental Results: VSWR 


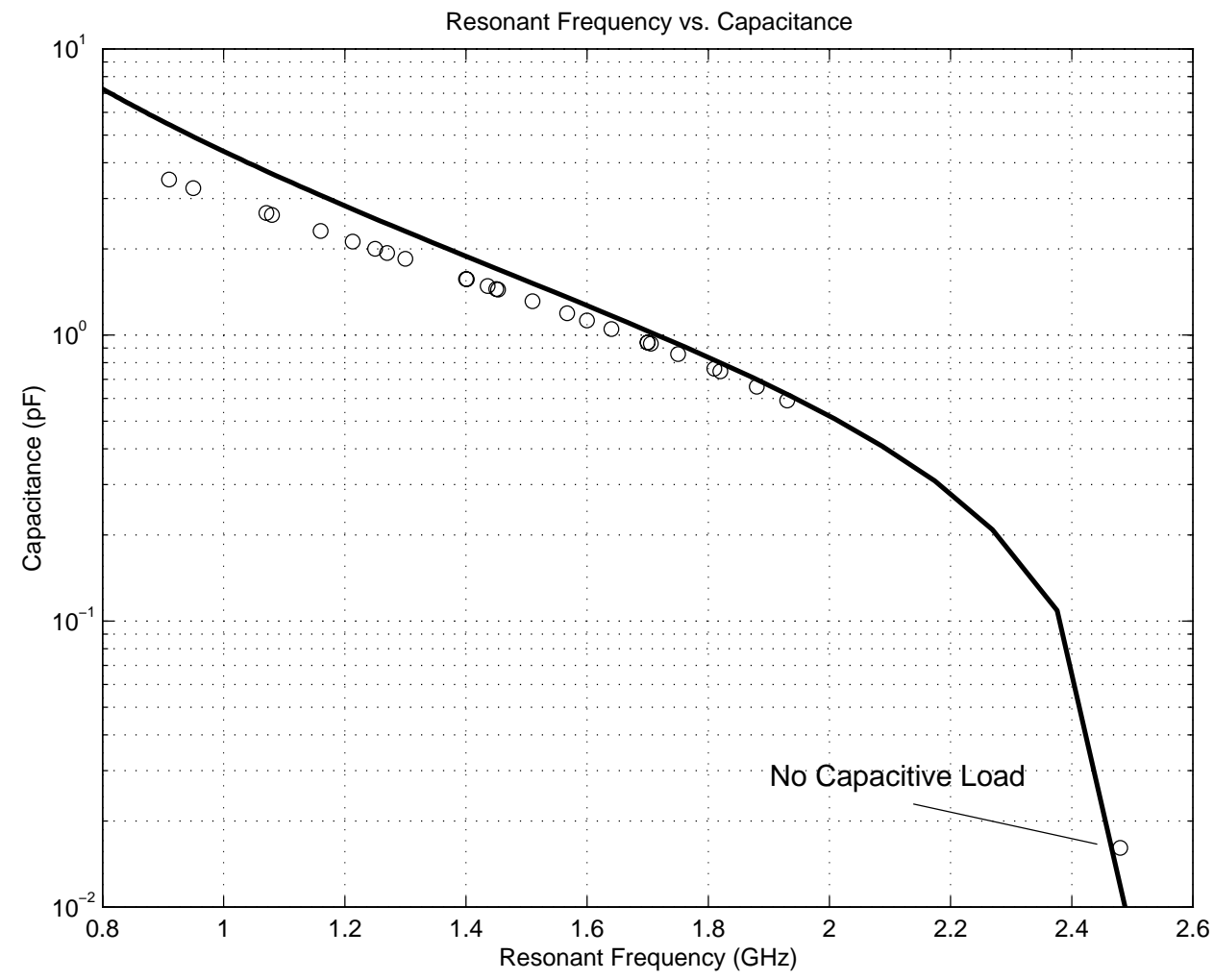

Fig. 11. The resonant frequency as a function of capacitance. The antenna geometry is: length $=25 \mathrm{~mm}$, width $=10 \mathrm{~mm}$, height $=5 \mathrm{~mm}$, offset $=7 \mathrm{~mm}$. Resonance without the capacitive load is $2.5 \mathrm{GHz}$.

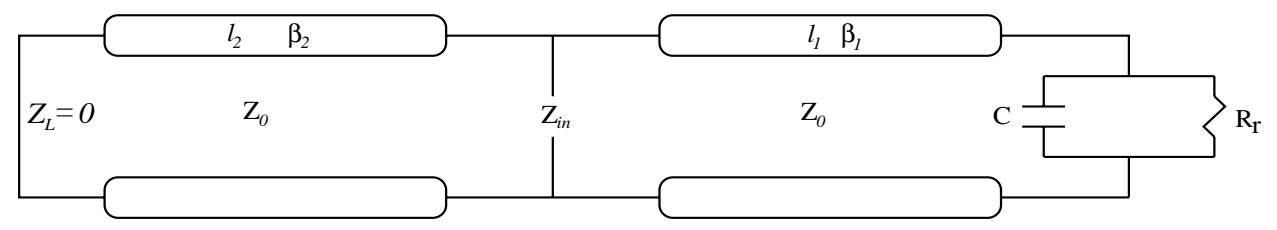

Fig. 12. Transmission Line Model of Capacitive Loaded Microstrip 